\title{
A HOMOTOPY THEORETIC PROOF OF THE ADDITIVITY OF THE TRACE
}

\author{
M. BREUER
}

Abstract. Murray and von Neumann gave in [10] an explicit definition of the trace function of a finite von Neumann algebra $\mathscr{M}$ in terms of a Riemann-Stieltjes integral. For this definition one only needs the spectral theorem for hermitian elements of $\mathscr{M}$ and the (relative) dimension function on the projections of $\mathscr{M}$. Although such a definition of the trace is very natural, the proof of the additivity of the trace is surprisingly long and unnatural. In the present paper results on the homotopy theory of unitary group $\mathfrak{A}$ of $\mathscr{M}$ are used to obtain a new proof of the additivity.

The periodicity theorem of $K$-theory relative to $\mathscr{M}$ which is proved in [5] without using a trace functioncan be used together with some standard techniques of homotopy theory to determine the stable homotopy of $\mathfrak{A}$. We need only the first stable homotopy group $\pi_{1}\left(\mathfrak{A}_{\infty}\right)$ of $\mathfrak{A}$ for the additivity proof. This group is isomorphic to the index group $I(\mathscr{M})$ which is also the range of the trace function $\varphi$ when restricted to the hermitian elements of $\mathscr{M}$. In the factorial case Araki- B. Smith- L. Smith [1] computed $\pi_{1}(\mathfrak{H})$ which turns out to be isomorphic to $\pi_{1}\left(\mathfrak{A}_{\infty}\right)$. We cannot use the method of [1] because it depends on the additivity of the trace function.

For a positive $S \in \mathscr{M}$ we consider an appropriate sequence $\left(S_{k}\right)$ approximating $S$ in the norm such that there is a sequence $\left(n_{k}\right)$ of integers satisfying $\exp \left(2 \pi \operatorname{in}_{k} S_{k}\right)=\mathrm{I}$. Then $\tau_{k}(t)=\exp \left(2 \pi i i_{k} t S\right)$, $0 \leqq t \leqq 1, k=1,2, \ldots$, is a sequence of loops in $\mathfrak{A}$ such that

$$
\varphi(S)=\lim \frac{1}{n_{k}}\left[\tau_{k}\right]_{\infty},
$$

where []$_{\infty}$ denotes the stable homotopy class. Elementary homotopy properties of loops in $\mathfrak{A}$ and simple density arguments together with the norm continuity of $\varphi$ then yield the additivity of $\varphi$. The norm continuity of $\varphi$ is not proved in this paper, because simple proofs of this property of $\varphi$ can be found in the literature e.g., Murray- von Neumann, loc.cit. To avoid topologizing the index group and other rather technical difficulties we assumed in the second part of this paper that $\mathscr{M}$ is a factor of type $\mathrm{II}_{1}$. Then $I(\mathscr{M})=\mathbf{R}$ and the usual topology of $\mathbf{R}$ can be taken.

An interesting question seems to be whether the proof of the periodicity theorem of [5] generalizes to $A W^{*}$-algebras. If so, the method of proving the existence of a trace used in this paper may also yield an existence proof of the trace in finite $A W^{*}$-algebra $\mathscr{M}$ with values in the index group $\mathrm{I}(\mathscr{M})$.

Received by the editors on April 13, 1978.

Copyright (C) 1980 Rocky Mountain Mathematics Consortium 
1. Review of $K_{\mathscr{r}}$-theory. Let $\mathscr{N}$ be a properly infinite and semifinite von Neumann algebra of operators of the complex Hilbert space $H$. The lattice of all projections of $\mathscr{N}$ is denoted by $P(\mathscr{N})$, the sublattice of finite projections by $P_{f}(\mathscr{N})$. For $E, F$ in $P(\mathscr{N})$ let

$$
J(E, F)=\left\{U \in \mathscr{N} \mid E=U^{*} U, F=U U^{*}\right\},
$$

Equip $J(E, F)$ with the norm topology.

Let $\xi$ be a vector bundle with compact base space $X$ and projection $p$ : $\xi \rightarrow X$. Let $\xi_{x}=p^{-1}(x)$ for $x \in X$. Suppose that the following data are given.

(I) Each $\xi_{x}$ is a complex Hilbert space.

(II) There is an open cover $\left\{X_{i}\right\}_{i \in I}$ of $X$ and a family $\left\{E_{i}\right\}_{i \in I}$ in $P(\mathscr{N})$ and an atlas $\left\{\varphi_{i}, X_{i}\right\}_{i \in I}$ of $\xi$ such that

$$
\varphi_{i}: p^{-1}\left(X_{i}\right) \rightarrow X_{i} \times E_{i}(H), i \in I .
$$

(III) Denote by $\varphi_{i, x}$ the restriction of $\varphi_{i}$ to $\xi_{x}$ for each $i \in I$ and $x \in X_{i}$. For $x \in X_{i} \cap X_{j}$ define the transition operator $g_{i j}(x): H \rightarrow H$ by

$$
g_{i j}(x)=\varphi_{i, x} \circ \varphi_{j, x}^{-1} \circ E_{j} .
$$

Then $x \rightarrow g_{i j}(x)$ is a continuous map of $X_{i} \cap X_{j}$ into $J\left(E_{i}, E_{j}\right)$.

We call a family of triples $\left\{\varphi_{i}, X_{i}, E_{i}\right\}_{i \in I}$ satisfying these conditions an $\mathscr{N}$-atlas of $\xi$. It is now clear how to define the structure of an $\mathscr{N}$-vector bundle.

Suppose that $\left\{\varphi_{i}, X_{i}, E_{i}\right\}_{i \in I}$ is an $\mathscr{N}$-atlas of $\xi$. If all $E_{i}$ are finite (resp. infinite), we say that $\xi$ has finite dimensional (resp. infinite dimensional) fibre relative to $\mathscr{M}$. It is clear how to define isomorphisms of $\mathscr{N}$-vector bundles (see [5]). Let $\operatorname{Vect}_{\mathcal{N}}(X)$ denote the set of all isomorphism classes of $\mathscr{N}$-vector bundles with finite fibre dimension and base space $X$. Given two $\mathscr{N}$-vector bundles $\xi$ and $\eta$ over $X$, one can always find $\mathscr{N}$-atlases of the form $\left\{X_{i}, \varphi_{i}, E_{i}\right\}_{i \in I}$ and $\left\{X_{i}, \phi_{i}, F_{i}\right\}_{i \in I}$ where $E_{i}$ is orthogonal to $F_{i}$ for each $i \in I$. Hence $\left\{X_{i}, \varphi_{i} \oplus \psi_{i}, E_{i}+F_{i}\right\}_{i \in I}$ is an $\mathscr{N}$-atlas of $\xi \oplus \eta$ defining the structure of an $\mathscr{N}$-vector bundle on $\xi \oplus \eta$. If the fibres of $\xi, \eta$ are finite dimensional, so are the ones of $\xi \oplus \eta$. Thus $\oplus$ defines the structure of a commutative monoid on $\operatorname{Vect}_{\mathcal{N}}(X)$. The universal group of this monoid is denoted by $K_{\mathcal{N}}(X)$. The canonical image of $\xi \in \operatorname{Vect}_{\mathcal{N}}(X)$ in $K_{\mathcal{N}}(T)$ is denoted by $[\xi]_{\mathcal{N}}$. For $E \in P_{f}(\mathcal{N})$ let $\Theta_{E, X}=X \times E(H)$ be the trivial $\mathscr{N}$-vector bundle with fibre $E(H)$. If $X$ is a one-point space, say $\left\{x_{0}\right\}$, then $K_{\mathcal{N}}\left(\left\{x_{0}\right\}\right)$ is denoted by $I(\mathcal{N})$ and called the index group of $\mathscr{N}$. The canonical map

$$
\operatorname{Dim}: P_{f}(\mathscr{N}) \rightarrow I(\mathscr{N})
$$

defined by 
$\operatorname{Dim} E::=\left[\Theta_{\left.E, x_{0}\right]}\right]_{\mathcal{r}}$

is a formal dimension function.

Composing Dim with an appropriate embedding of $I(\mathscr{N})$ into the centre of $\mathscr{N}$ one obtains von Neumann's relative dimension function (with values in the positive elements of the centre). In what follows the formal dimension function is sufficient. Observe that $I(\mathscr{N})=\mathbf{R}$ if and only if $\mathscr{N}$ is a factor of type II.

In the following let $X$ be locally compact and $X^{+}=X \cup\{+\}$ be its one-point compactification. If $\mathfrak{a}$ is a complex vector bundle over $X^{+}$with finite dimensional fibre in the usual sense, and if $\xi$ is an $\mathscr{N}$-vector bundle with finite dimensional fibre, then their tensor product $\mathfrak{a} \otimes \xi$ is an $\mathscr{N}$ vector bundle, whose fibre is again finite dimensional (relative to $\mathscr{N}$ ). This gives rise to a pairing

$$
K\left(X^{+}\right) \times K_{\mathcal{N}}\left(X^{+}\right) \rightarrow K_{\mathscr{N}}\left(X^{+}\right)
$$

which gives $K_{\mathscr{N}}\left(X^{+}\right)$the structure of a $K\left(X^{+}\right)$-module. Using this structure one can define an external product

$$
K\left(X^{+}\right) \times K_{\mathscr{N}}\left(Y^{+}\right) \rightarrow K_{\mathscr{N}}\left(X^{+} \times Y^{+}\right),
$$

where $X, Y$ are locally compact spaces. As in $K$-theory there is a direct sum decomposition

$$
K_{\mathscr{N}}\left(X^{+}\right)=I(\mathscr{N}) \oplus \tilde{K}_{\mathscr{N}}\left(X^{+}\right)
$$

of the abelian group $K_{\mathscr{H}}\left(X^{+}\right)$. This defines the reduced functor $\tilde{K}_{\mathscr{N}}$. Define

$$
K_{\mathscr{N}}(X):=\tilde{K}_{\mathscr{N}}\left(X^{+}\right), X \text { locally compact. }
$$

$K_{\mathscr{S}}$ is a functor of the category of locally compact spaces and proper continuous maps. It is easy to see that $K_{\mathscr{N}}(X)$ is a submodule of the $K\left(X^{+}\right)$module $K_{\mathcal{N}}\left(X^{+}\right)$. Using standard techniques of $K$-theory (see Atiyah [2]) it can be shown that the external product induces a pairing

$$
K(X) \times K_{\mathscr{N}}(Y) \rightarrow K_{\mathscr{N}}(X \times Y),
$$

where $X, Y$ are again locally compact.

Let $\gamma_{n}$ be the complex line bundle over $S^{2}=\left(\mathbf{R}^{2}\right)^{+}$arising from the clutching function $z^{n}: S^{1} \rightarrow S^{1}$. Let $\mathfrak{b} \in K\left(S^{2}\right)$ be the Bott class which is defined by

$$
\mathfrak{b}:=\left[\gamma_{1}\right]-\left[\gamma_{0}\right]
$$

It is well-known (and quite obvious) that $\mathfrak{b}$ is contained in the subgroup $K\left(\mathbf{R}^{2}\right)$ of $K\left(S^{2}\right)$. For $X$ locally compact define

$$
\beta: K_{\mathscr{N}}(X) \rightarrow K_{\mathscr{N}}\left(\mathbf{R}^{2} \times X\right)
$$


by $\beta\left(|\xi|_{\mathcal{N}}\right):=b \cdot[\xi]_{\mathcal{N}}$ (external product).

THEOREM 1. For every locally compact space $X$ the map $\beta$ is an isomorphism of the abelian group $K_{\mathscr{N}}(X)$ onto $K_{\mathscr{N}}\left(\mathbf{R}^{2} \times X\right)$.

If $\mathscr{N}$ is the algebra of all bounded operators of $H$, then $K_{\mathscr{N}}(X)=K(X)$. In that case theorem 1 reduces to the periodicity theorem of $K$-theory. The general case has been proved in [5].

2. The stable homotopy of the unitary group of a finite von Neumann algebra. Let $\mathfrak{n}$ be the two-sided ideal of $\mathscr{N}$ generated by $P_{f}(\mathscr{N})$. The elements of $\mathfrak{n}$ are called compact. Call $T \in \mathscr{N}$ Fredholm if $T$ is regular modulo $\mathfrak{n}$. $\mathscr{F} \mathscr{N}$ denotes the monoid of Fredholm elements of $\mathscr{N}$ equipped with the norm topology. Let $X$ be locally compact. $\left[X^{+}, \mathscr{F} \mathscr{N}\right]$ denotes the monoid of homotopy classes of continuous maps of $X^{+}$into $\mathscr{F} \mathscr{N}$; and $[X, \mathscr{F} \mathscr{N}]_{c}$ be the submonoid of all proper maps of $X$ into $\mathscr{F} \mathscr{N}$ mapping + onto the unit element $I$ of $\mathscr{N}$. As in Atiyah [2, Appendix] or Janich [9] one can associate to each $[f] \in\left[X^{+}, \mathscr{F} \mathscr{N}\right]$ an element index $f \in K_{\mathcal{N}}\left(X^{+}\right)$.

THEOREM 2. For each locally compact space $X$ the map

$$
\text { index: }\left[X^{+}, \mathscr{F} \mathscr{N}\right] \rightarrow K_{\mathscr{N}}\left(X^{+}\right)
$$

is an isomorphism. Under this isomorphism $[X, \mathscr{F} \mathscr{N}]_{c}$ is mapped onto $K_{\mathcal{N}}(X)$ If $\mathscr{N}$ is the algebra $\mathscr{L}(H)$ of all bounded operators of the complex infinite dimensional Hilbert space $H$, theorem 2 reduces to a well-known theorem of Atiyah and Jänich (loc. cit.). The general case has been proved in [5].

THEOREM 3. The homotopy groups of $\mathscr{F} \mathcal{N}$ are

$$
\pi_{n}(\mathscr{F} \mathscr{N})=\left\{\begin{array}{cl}
I(\mathscr{N}) & \text { for } n \text { even } \\
0 & \text { for } n \text { odd } .
\end{array}\right.
$$

Sketch of Proof. Taking $X=\mathbf{R}^{n}$, theorem 1 and 2 imply homotopy periodicity

$$
\pi_{n}(\mathscr{F} \mathscr{N})=\pi_{n+2}(\mathscr{F} \mathscr{N}) .
$$

In [5] it has been shown that

$$
\pi_{0}(\mathscr{F} \mathscr{N})=I(\mathscr{N}),
$$

It remains to determine the first homotopy group of $\mathscr{F} \mathscr{N}$. For $\alpha \in$ $I(\mathscr{N})$ let

$$
\operatorname{Gr}(\alpha)=\left\{E \in P_{f}(\mathscr{N}) \mid \operatorname{Dim} E=\alpha\right\} .
$$

Equip $\operatorname{Gr}(\alpha)$ with the norm topology (note that $\operatorname{Gr}(\alpha)$ is empty or a connected component of $\left.P_{f}(\mathscr{N})\right)$. In [5] it has been shown that $\operatorname{Gr}(\alpha)$ is a classifying space for $\mathscr{N}$-vector bundles over compact spaces with fibre 
dimension $\alpha$. Moreover $\operatorname{Gr}(\alpha)$ is simply connected (See [4]). Hence every $\mathscr{N}$-vector bundle over $S^{1}$ is trivial. This implies that $\mathscr{F} \mathscr{N}$ is simply connected.

For any $C^{*}$-algebra $A$ let $\mathscr{G} A$ denote the group of regular elements of $A$ equipped with the norm topology, and $\mathscr{G}_{0} A$ be the connected component of $\mathscr{G} A$ containing the unit element of $A$.

Let $\mathscr{G}(I+\mathfrak{n})$ be the subgroup of $\mathscr{G N}$ consisting of the elements of the form $I+T$ where $T$ is compact and 1 not in the spectrum of $T$. The canonical map of $\mathscr{N}$ onto $\mathscr{N} / \mathfrak{n}$ induces a map

$$
\kappa: \mathscr{F} \mathscr{N} \rightarrow \mathscr{G}(\mathscr{N} / \mathfrak{n})
$$

which is a homotopy equivalence. In addition $\kappa$ induces a surjective group homomorphism

$$
\pi: \mathscr{G} \mathscr{N} \rightarrow \mathscr{G}_{0}(\mathscr{N} / \mathfrak{n})
$$

which is a fibration with typical fibre $\mathscr{G}(I+\mathfrak{n})$. Using the generalized Kuiper theorem for properly infinite von Neumann algebras (see [4] or [7]) the exact homotopy sequence of this fibration yields

THEOREM 4. The homotopy groups of $\mathscr{G}(I+\mathfrak{n})$ are

$$
\pi_{n} \mathscr{G}(I+\mathfrak{n})=\left\{\begin{array}{l}
0 \quad \text { for } n \text { even } \\
I(\mathscr{N}) \text { for } n \text { odd } .
\end{array}\right.
$$

Since $\mathscr{N} \subseteq \mathscr{L}(H)$ is properly infinite and semifinite, there are Hilbert spaces $L, l^{2}$ and a finite von Neumann algebra $\mathscr{M} \subseteq \mathscr{L}(L)$ such that

$$
H=L \otimes l^{2}
$$

(Hilbert space tensor product)

and

$$
\mathscr{N}=\mathscr{M} \otimes \mathscr{L}\left(l^{2}\right) \quad\left(W^{*} \text {-tensor product }\right)
$$

We also can assume that $l^{2}$ is separable. Let $\left(e_{n}\right)_{n=1,2, \ldots}$ be an increasing sequence of projections in $\mathscr{L}\left(l^{2}\right)$ such that $\operatorname{Dim}\left(e_{n}\right)=2^{n}$ and the supremum of this sequence is $I_{2}$, the unit element of $\mathscr{L}\left(l^{2}\right)$. Denote the unit element of $\mathscr{M}$ by $I_{\mathscr{M}}$. Let $E_{n}:=I_{\mathscr{M}} \otimes e_{n}$ and $\mathscr{M}_{n}:=E_{n} \mathscr{N} E_{n}$. Observe that there are canonical isomorphisms $\mathscr{M}_{n} \cong \mathscr{M} \otimes \mathscr{L}\left(\mathbf{C}^{2 n}\right), n=1,2, \ldots$, in particular $\mathscr{M}_{1} \cong \mathscr{M}$. Since $E_{n}$ is finite, one has $\mathscr{M}_{n} \subseteq \mathfrak{n}$. Embed $\mathscr{G} \mathscr{M}_{n}$ in $\mathscr{G}(I+\mathfrak{n})$ by

$$
\iota_{n}(T):=T+\left(I-E_{n}\right), T \in \mathscr{G} \mathscr{M}_{n} .
$$

Obviously $\iota_{n} \mathscr{G} \mathscr{M}_{n}$ is contained in $\iota_{n+1} \mathscr{G} \mathscr{M}_{n+1}$. Equip

$$
\mathscr{G} \mathscr{M}_{\infty}:=\underline{\lim } \iota_{n} \mathscr{G} \mathscr{M}_{n}
$$

with the inductive limit topology. 
THEOREM 5. $\mathscr{G} \mathscr{M}_{\infty}$ is homotopy equivalent to $\mathscr{G}(I+\mathfrak{n})$. Hence

$$
\pi_{n}\left(\mathscr{G} \mathscr{M}_{\infty}\right)= \begin{cases}0 & \text { for } n \text { even } \\ I(\mathscr{M}) & \text { for } n \text { odd }\end{cases}
$$

Observe that $\mathscr{M}$ and $\mathscr{N}$ have the same index group. If $\mathscr{N}$ is the full operator algebra $\mathscr{L}(H)$, then $\mathfrak{n}$ is the two-sided ideal of compact operators in the usual sense. In that case theorem 5 reduces to a result of Breuer-Cordes [6] and Palais [11]. The proofs of [6] and [11] do not translate verbally, because in the general case $\bigcup_{m=1}^{\infty} \mathscr{M}_{m}$ is not dense in $\mathfrak{n}$.

Proof of THeORem 5. More exactly we show that the inclusion map c: $\mathscr{G} \mathscr{M}_{\infty} \rightarrow \mathscr{G}(I+\mathfrak{n})$ is a homotopy equivalence. Since the inductive limit topology is finer than the norm topology, $\iota$ is continuous. To show that $\iota$ induces a surjective map $\pi_{n}\left(\mathscr{G} \mathscr{M}_{\infty}\right) \rightarrow \pi_{n}(\mathscr{G}(I+\mathfrak{n}))$ for each $n$ it suffices to show that each continuous map $f$ of a compact space $X$ into $\mathscr{G}(I+\mathfrak{n})$ is homotopic to a map of $X$ into $\mathscr{G}\left(\mathscr{M}_{\infty}\right)$. Given such a map $f$ there is a sequence $\left(F_{m}\right)_{m=1,2, \ldots}$ in $P_{f}(\mathscr{N})$ satisfying

$$
F_{m} \leqq F_{m+1}, \sup F_{m}=I, \operatorname{Dim} F_{m}=\operatorname{Dim} E_{m} \text { for } m=1,2, \ldots
$$

and

$$
\lim \left\|f(x)-f(x) F_{m}\right\|=0, \lim \left\|f(x)-F_{m} f(x)\right\|=0
$$

uniformly on $X$. Then there is a unitary element $U$ of $\mathscr{N}$ such that $U^{*} E_{m} U=F_{m}$ for all $m$. Since the unitary group of $\mathscr{N}$ is connected, there is a continuous path $U_{t}, 1 \leqq t \leqq 1$, in the unitary group of $\mathscr{N}$ such that $U_{1}=U$ and $U_{0}=I$. Define $f_{t}=U_{t}^{*} f U_{t}, 0 \leqq t \leqq 1$. Then $f_{1}$ satisfies $f_{1}(X) \subset \mathscr{G}(I+\mathfrak{l})$ and

$$
\lim \left\|f_{1}(x)-f_{1}(x) E_{m}\right\|=0, \lim \left\|f_{1}(x)-E_{m} f_{1}(x)\right\|=0
$$

uniformly on $X$. For $m$ sufficiently large the segment

$$
f_{1+t}(x)=(1-t) f_{1}(x)+t E_{m} f_{1}(x) E_{m}, x \in X, 0 \leqq t \leqq 1
$$

lies completely in $\mathscr{G}(I+\mathfrak{n})$. Hence $f_{t}, 0 \leqq t \leqq 2$, is a homotopy in $\mathscr{G}(I+\mathfrak{n})$ connecting $f=f_{0}$ with the map $f_{2}$ of $X$ into $\mathscr{G}\left(\mathscr{M}_{\infty}\right)$.- -To show that $c$ induces an injective map $\pi_{n}\left(\mathscr{G} \mathscr{M}_{\infty}\right) \rightarrow \pi_{n}(\mathscr{G}(I+\mathfrak{n}))$ for all $n$ let $f$, $g$ be two continuous maps of $X$ into $\mathscr{G} \mathscr{M}_{\infty}$. We have to show that if $f$, $g$ are homotopic within $\mathscr{G}(I+\mathfrak{l})$ they are homotopic within $\mathscr{G} \mathscr{M}_{\infty}$. Let $Y=X \times[0,1]$ and $h: Y \rightarrow \mathscr{G}(I+\mathfrak{n})$ be such that $h(x, 0)=f(x), h(x, 1)=$ $g(x)$ for all $x \in X$. Since $X$ is compact and $\mathscr{G} \mathscr{M}_{\infty}$ is a strict inductive limit, there is an $n$ such that $f, g$ map $X$ into $\iota_{n} \mathscr{G}\left(\mathscr{M}_{n}\right)$. Choose a sequence $\left(G_{k}\right)$ in $P_{f}(\mathscr{N})$ satisfying

$$
G_{1}=E_{n}, \operatorname{Dim} G_{k}=\operatorname{Dim} E_{n k}, G_{m} \leqq G_{m+1}, \sup _{k} G_{k}=I
$$


and

$$
\lim \left\|h(y)-h(y) G_{k}\right\|=0, \lim \left\|h(y)-G_{k} h(y)\right\|=0
$$

uniformly on $Y$. Let $V$ be a unitary element of the reduced algebra $\left(I-E_{n}\right) \mathcal{N}\left(I-E_{n}\right)$ such that $V^{*}\left(E_{n k}-E_{n}\right) V=G_{k}-G_{1}$. Then $W=V+E_{n}$ is unitary in $\mathscr{N}$ such that $W^{*} E_{n} W=E_{n}$, and consequently $W^{*} f(x) W=$ $f(x), W^{*} g(x) W=g(x)$ for all $x \in X$. Define

$$
\tilde{h}(y):=W^{*} h(y) W \text { for all } y \in Y,
$$

Then $h$ is still a homotopy between $f$ and $g$. It follows from the construction of $\tilde{h}$ that

$$
\lim \left\|\tilde{h}(y)-\tilde{h}(y) E_{m}\right\|=0, \lim \left\|\tilde{h}(y)-E_{m} \tilde{h}(y)\right\|=0
$$

uniformly on $Y$. Choosing $m$ sufficiently large $h(y):=E_{m} \tilde{h}(y) E_{m}$ is a homotopy between $f$ and $g$ within $\iota_{m} \mathscr{G}\left(\mathscr{U}_{m}\right) \subset \mathscr{G}\left(\mathscr{M}_{\infty}\right)$.

Let $\mathfrak{A}$ be the group $f$ unitary elements of $\mathscr{M} ; \mathfrak{A}_{n}$ be the group of unitary elements in $\iota_{n} \mathscr{G} . \|_{n}$. Let $U(n)$ be the group of unitary $(n, n)$-matrices. Then there is a canonical isomorphism

$$
\mathfrak{A}_{n} \cong \mathfrak{H} \otimes U\left(2^{n}\right) .
$$

The inclusion $\iota_{n} \mathscr{G} \mathscr{M}_{n} \subseteq \iota_{n+1} \mathscr{G} \mathscr{M}_{n+1}$ induces an inclusion $\mathfrak{U}_{n} \subseteq \mathfrak{A}_{n+1}$ which is compatible with the usual inclusion $U(m) \subseteq U(m+h)$ via the above tensor product decomposition taking $\left.m=k=2^{n}\right)$. The polar decomposition lemma implies that the subgroup

$$
\mathfrak{A}_{\infty}=\lim \mathfrak{A}_{n}
$$

equipped with the inductive limit topology is homotopy equivalent to $\mathscr{G} \mathscr{M}_{\infty}$. Hence we have

THEOREM 6. The homotopy groups of $\mathfrak{A}_{\infty}$ are

$$
\pi_{n}\left(\mathfrak{U}_{\infty}\right)= \begin{cases}0 & \text { for } n \text { even } \\ I(\mathscr{M}) & \text { for } n \text { odd }\end{cases}
$$

In the following we identify the unitary group $\mathfrak{A}$ of $\mathscr{M}$ with the subgroup $\mathfrak{A}_{1}$ of $\mathfrak{A}_{\infty}$, omitting the map c. If $f$ is a map of some space into $\mathfrak{A}$, then $[f]$ resp. $[f]_{\infty}$ denotes the homotopy class of $f$ as a map into $\mathfrak{A}$ resp. $\mathfrak{A}_{\infty}$.

3. Definition of the trace in terms of homotopy classes of loops. Henceforth we assume that $\mathscr{U}$ is a factor of type $\mathrm{II}_{1}$. Choose an isomophism of the index group $I(\mathscr{M})$ onto $\mathbf{R}$ by mapping the unit element of $\mathscr{M}$ onto $1 \in \mathbf{R}$. Using this isomophism we write $I(\mathscr{M})=\mathbf{R}$. Let $\mathscr{M}_{h}$ resp. $\mathscr{M}_{+}$ denote the set of hermitian resp. positive elements of $\mathscr{M}$. One possible definition of the trace function 


$$
\varphi: \mathscr{M}_{h} \rightarrow \mathbf{R}
$$

is as follows. Let $S \in \mathscr{M}_{h}$ and

$$
S=\int \lambda d E_{\lambda}
$$

be its spectral representation. Then

$$
\varphi(S)=\int \lambda d\left(\operatorname{Dim} E_{\lambda}\right)
$$

where the integral is a Riemann-Stieltjes integral. The advantage of this definition is that one does not need to give an existence proof; the disadvantage is that the proof of the additivity is difficult. We give a homotopy theoretic interpretation of $\varphi$ in order to obtain a more natural proof of the additivity.

For $S \in \mathscr{M}_{k}$ and $n \in \mathbf{Z}$ define the path

$$
\tau_{n, S}:[0,1] \rightarrow \mathfrak{A}
$$

by

$$
\tau_{n, S}(t)=\exp (2 \pi \text { int } S), 0 \leqq t \leqq 1,
$$

Instead of $\tau_{1, S}$ we write $\tau_{S}$. If the spectrum of $n S$ is contained in $\mathbf{Z}$, then $\tau_{n, S}$ is a loop attached at the unit element $I$ of $\mathfrak{U}$. The following theorem gives a homotopy theoretic interpretation of the dimension function.

Theorem 7. Let $E, F$ be projections in $\mathscr{M}$. Then $\operatorname{Dim} E=\operatorname{Dim} F$ if and only if the loops $\tau_{E}$ and $\tau_{F}$ and homotopic in $\mathfrak{A}$.

Proof. Suppose that $\operatorname{Dim} E=\operatorname{Dim} F$. Then there is a $U \in \mathfrak{A}$ such that $U^{*} E U=F$. Since $\mathfrak{A}$ is connected, there is a continuous path $U_{s}, 0 \leqq$ $s \leqq 1$, such that $U_{0}=I$ and $U_{1}=U$. Define $G_{s}=U_{s}^{*} E U_{s}$. Then $\tau_{G_{s}}$, $0 \leqq s \leqq 1$, is a continuous deformation of $\tau_{E}$ into $\tau_{F}$ within $\mathfrak{A}$ such that $\tau_{G_{s}}(0)=I$. To prove the converse it suffices to show that $\left[\tau_{E}\right]=0$ implies $\operatorname{Dim} E=0$. Let $\xi_{n}$ be the $\mathcal{N}$-vector bundle with fibre $L$ and base space $S^{2}$ arising from the map $\tau_{n, E}: S^{1} \rightarrow \mathfrak{A}$ via the clutching construction. Since

$$
\exp (2 \pi \operatorname{int} E)=(\exp (2 \pi \mathrm{int})) E+(I-E)
$$

one easily sees that

$$
\xi_{n}=\gamma_{n} \otimes E(L) \otimes \Theta_{I-E, S^{2}}
$$

Hence

$$
\left[\xi_{-1}\right]_{\mathscr{N}}-\left[\xi_{0}\right]_{\mathscr{r}}=\mathfrak{b} \cdot \operatorname{Dim} E,
$$

where $\mathfrak{b}$ is the Bott class defined in 1 . Since $\left[\tau_{n, E}\right]=n \cdot\left[\tau_{E}\right]=0$, one has $\left[\xi_{n}\right]_{\mathcal{V}}=0$. Hence $\mathfrak{b} \cdot \operatorname{Dim} E=0$ which implies Dim $E=0$ 
Corollary 1. Dim $E=\operatorname{Dim} F$ if and only if the loops $\tau_{E}$ and $\tau_{F}$ are homotopic in $\mathfrak{A}_{\infty}$.

Proof. If $\operatorname{Dim} E=\operatorname{Dim} F$, then $\left[\tau_{E}\right]=\left[\tau_{F}\right]$ according to Proposition 1 . Then also $\left[\tau_{E}\right]_{\infty}=\left[\tau_{F}\right]_{\infty}$ because each homotopy in $\mathfrak{A}$ is a homotopy in $\mathfrak{A}_{\infty}$. Suppose conversely that $\left[\tau_{E}\right]_{\infty}=\left[\tau_{F}\right]_{\infty}$. Then $\tau_{E}$ and $\tau_{F}$ are homotopic in some $\mathfrak{A}_{n}$. It follows from Proposition 1 that $E$ and $F$ have the same dimension relative to $\mathscr{M}_{n}$. This means that $E, F$ are equivalent projections in $\mathscr{M}_{n}$. Then $E, F$ are also equivalent in every reduced subalgebra containing $E$ and $F$. Since $\mathscr{M}$ is a reduced subalgebra of $\mathscr{M}_{n}$ it follows that $E, F$ are equivalent in $\mathscr{M}$, hence $\operatorname{Dim} E=\operatorname{Dim} F$.

Corollary 2. Suppose that $E F=0$. Then

$$
\left[\tau_{E+F}\right]_{\infty}=\left[\tau_{E}\right]_{\infty}+\left[\tau_{F}\right]_{\infty} .
$$

Hence there is an isomorphism of $I(\mathscr{M})$ onto $\pi_{1}\left(\mathfrak{A}_{\infty}\right)$ mapping Dim $E$ onto $\left[\tau_{E}\right]_{\infty}$. We may therefore write $I(\mathscr{M})=\pi_{1}\left(\mathfrak{A}_{\infty}\right)=\mathbf{R}$, in particular $\operatorname{Dim} I=\left[\tau_{I}\right]_{\infty}=1$.

Proof. Using the addition theorem of the exponential function one obtains

$$
\left[\tau_{E+F}\right]_{\infty}=\left[\tau_{E} \cdot \tau_{F}\right]_{\infty}=\left[\tau_{E}\right]_{\infty}+\left[\tau_{F}\right]_{\infty}
$$

Using this and the universal property of the index group $I(\mathscr{M})=K\left(\left\{x_{0}\right\}\right)$ one obtains a homomorphism of $I(\mathscr{M})$ into $\pi_{1}\left(\mathfrak{A}_{\infty}\right)$. Corollary 1 implies that this homomorphism is injective. It is also surjective because $\pi_{1}\left(\mathfrak{H}_{\infty}\right)$ is isomorphic to $\mathbf{R}$ by theorem 6. It is obvious that $\operatorname{Dim} I$ is mapped onto $\left[\tau_{I}\right]_{\infty}$.

COROLlary 3. Let $S \in \mathscr{M}_{h}$ be of the form

$$
S=q_{1} E_{1}+\cdots+q_{m} E_{m}
$$

where $E_{1}, \ldots, E_{m}$ are pairwise orthogonal projections of $\mathscr{M}$ and $q_{1}, \ldots, q_{m}$ are rational numbers with smallest common denominator $c$. Then

$$
\varphi(S)=\frac{1}{c}\left[\tau_{c, S}\right]_{\infty}
$$

Proof. Using the addition theorem of the exponential function this is proved in a manner similar to the proof of corollary 2 .

CoRollary 4. Let $S \in \mathscr{M}_{h}$ and let $A$ be a commutative $W^{*}$-subalgebra of $\mathscr{M}$ containing $S$. Let $\left(S_{k}\right)_{k=1,2, \ldots}$ be a sequence in $A_{h} \subset \mathscr{M}_{h}$ such that $S_{k}=$ $q_{k, 1} E_{k, 1}+\cdots+q_{k, m_{k}} E_{k, m_{k}}$, where $E_{k, j}, j=1, \ldots, m_{k}$ are pairwise orthogonal projections in $A$ for each $k$, and where $q_{k, j}, j=1,2, \ldots$ are rational 
numbers with smallest common denominator $c_{k}, k=1,2, \ldots$. Suppose that $\lim \left\|S-S_{k}\right\|=0$. Then

$$
\varphi(S)=\lim _{k \rightarrow \infty} \frac{1}{c_{k}}\left[\tau_{c_{k}, S k}\right]_{\infty} .
$$

Proof. This follows easily from corollary 3 and the continuity of the restriction of $\varphi$ to $A_{h}$.

4. Proof of the additivity of $\varphi$. Define the following subspaces of $\mathscr{M}$ resp. $\mathscr{M}_{+} \times \mathscr{M}_{+}$

$$
\begin{aligned}
& \mathscr{E}=\left\{S / S \in \mathscr{M}_{+} \text {and }\|\exp (2 \pi i S)-\mathrm{I}\|<2\right\}, \\
& I I=\{(S, T) / S, T \text { and } S+T \text { are elements of } \mathscr{E}\} .
\end{aligned}
$$

For each $S \in \mathscr{E}$ define

$$
\mathscr{E}_{S}=\left\{T / T \in \mathscr{M}_{+} \text {and } S_{+} T \in \mathscr{E}\right\}
$$

and

$$
\mathscr{F}_{s}=\{T /(S, T) \in \Pi\} .
$$

Lemma 1. The set $\mathscr{E}$ is open and dense in $\mathscr{U}_{+}$in the norm topology.

Proof. This is a straightforward application of functional calculus. The details are as follows. Let $\mathfrak{A}_{0}=\{U \in \mathfrak{2} /\|U-I\|<2\}$. Then $\mathscr{E}$ is the inverse image of $\mathfrak{A}_{0}$ under the map $T \mapsto \exp (2 \pi i T), T \in \mathscr{M}_{+}$. Since $\mathfrak{A}_{0}$ is open in $\mathfrak{A}$ and exp is continuous, $\mathscr{E}$ is open in $\mathscr{M}_{+}$. Let $T \in \mathscr{M}_{+}$and $\|\exp (2 \pi i T)-I\|=2$. We have to show that for each $\delta>0$ there is a $T_{\delta} \in \mathscr{E}$ satisfying $\left\|T-T_{\delta}\right\|<\delta$. Let $A$ be a commutative $W^{*}$-subalgebra of $\mathscr{U}$ containing $T$. We identify $A$ with the algebra of all continuous complexvalued functions on the maximal ideal space $\Omega$ of $A$ via the Gelfand isomorphism. The norm of $A$ is then the sup-norm of functions on $\Omega$. Since $\Omega$ is compact and completely disconnected, there are a finite open cover $N_{1}, \ldots, N_{m}$ of $\Omega$ and functions $f_{1}, \ldots, f_{m} \in A_{+}$such that the following holds

1) $N_{i} \cap N_{j}=\varnothing$ for $i \neq j$,

2) $\left|\exp \left(2 \pi \sqrt{-1} f_{i}(\omega)\right)-1\right|<2$ for $\omega \in N_{i}$,

3) $f_{i}(\omega)=0$ for $\omega \notin N_{i}$, and

4) $\left|f_{i}(\omega)-T(\omega)\right|<\delta$ for $\omega \in N_{i}$.

Then $T:=f_{1}+\cdots+f_{m}$ satisfies the required conditions.

Lemma $2 \mathscr{F}_{s}$ is open and dense in $\mathscr{M}_{+}$in the norm topology.

Proof. It is easy to see that $\mathscr{E}_{S}$ is open and dense in $\mathscr{M}_{+}$. One has $\mathscr{F}_{S}=\mathscr{E} \cap \mathscr{E}_{S}$. Hence $\mathscr{F}_{S}$ is open and dense in $\mathscr{M}_{+}$. 
Definition. For each $S \in \mathscr{E}$ let $Q_{S}$ be the unique hermitian element of $\mathscr{M}$ satisfying

$\left\|Q_{S}\right\|<1 / 2$ and $\exp \left(2 \pi i Q_{S}\right)=\exp (2 \pi i S)$.

For each $S \in \mathscr{E}$ let $\sigma_{S}$ be the closed loop

$$
\sigma_{S}(t)= \begin{cases}\exp (4 \pi i t S) & \text { for } 0 \leqq t \leqq 1 / 2 \\ \exp \left(4 \pi i(1-t) Q_{S}\right) & \text { for } 1 / 2 \leqq t \leqq 1\end{cases}
$$

Lemma 4. For each $S \in \mathscr{E}$ one has

$$
\left[\sigma_{S}\right]_{\infty}=\varphi(S)-\varphi\left(Q_{S}\right) .
$$

PROOF. Using the spectral theorem we choose a sequence $\left(S_{k}\right)$ satisfying the hypotheses of corollary 4 of theorem 7. Since $\mathscr{E}$ is open and $S \in \mathscr{E}$ we may also assume $S_{k} \in \mathscr{E}$. Let $Q_{k}:=Q_{S_{k}}$ and define

$$
\omega_{k}(t)=\exp \left(2 \pi i c_{k} Q_{k}\right), 0 \leqq t \leqq 1
$$

Then $\omega_{k}(1)=\exp \left(2 \pi i c_{k} Q_{k}\right)=\exp \left(2 \pi i c_{k} S_{k}\right)=I$, so that $\omega_{k}$ is a loop at $I$. One easily verifies the following homotopy formula

$$
\left[\tau_{c_{k}, S_{k}}\right]=c_{k} \cdot\left[\sigma_{S_{k}}\right]+\left[\omega_{k}\right] .
$$

Since $\lim \left\|S-S_{k}\right\|=0$, one has $\left[\sigma_{S}\right]=\left[\sigma_{S_{k}}\right]$ for $k$ sufficiently large. Hence

$$
\left[\sigma_{S}\right]_{\infty}=\frac{1}{c_{k}}\left[\tau_{c_{k}, S_{k}}\right]_{\infty}-\frac{1}{c_{k}}\left[\omega_{k}\right]_{\infty} .
$$

Using corollary 3 of theorem 7 one obtains

$$
\left[\sigma_{S}\right]_{\infty}=\varphi\left(S_{k}\right)-\varphi\left(Q_{k}\right) .
$$

Using the norm continuity of $\varphi$ on commutative $W^{*}$-subalgebras of $\mathscr{M}$ and the relations $\lim \left\|S-S_{k}\right\|=0, \lim \left\|Q-Q_{k}\right\|=0$ one obtains lemma 3 from the last formula by taking the limit as $k \rightarrow \infty$.

Lemma 4. For all pairs $(S, T) \in$ II one has

$$
\varphi(S+T)-\varphi(S)-\varphi(T)=\varphi\left(Q_{S}+Q_{T}\right)-\varphi\left(Q_{S}\right)-\varphi\left(Q_{T}\right) .
$$

Proof. Since $S, T$ do not necessarily commute, we use the generalized addition theorem of the exponential function which is as follows (see Bourbaki [3, Chap. III §6 Prop. 8])

$$
\lim \left\|\exp 2 \pi i(S+T)-\left(\left(\exp \frac{2 \pi i}{n} S\right)\left(\exp \frac{2 \pi i}{n} T\right)\right)^{n}\right\|=0 .
$$

Since $S+T \in \mathscr{E}$ this formula implies that there is an $n_{0}$ such that $n \geq n_{0}$ implies 


$$
\left.\|\left(\exp \frac{2 \pi i}{n} S\right)\left(\exp \frac{2 \pi i}{n} T\right)\right)^{n}-I \|<2
$$

Let $P_{n}$ be the unique element of $\mathscr{M}_{h}$ satisfying $\left\|P_{n}\right\|<1 / 2$ and

$$
\left(\left(\exp \frac{2 \pi i}{n} S\right)\left(\exp \frac{2 \pi i}{n} T\right)\right)^{n}=\exp \left(2 \pi i P_{n}\right) \text {. }
$$

Define

$$
\rho_{S, T}(t)= \begin{cases}\left(\left(\exp \frac{4 \pi i}{n} t S\right)\left(\exp \frac{4 \pi i}{n} t T\right)\right)^{n} & \text { for } 0 \leqq t \leqq \frac{1}{2} \\ \exp \left(4 \pi i(1-t) P_{n}\right) & \text { for } \frac{1}{2} \leqq t \leqq 1\end{cases}
$$

and

$$
\nu_{n}(t)= \begin{cases}\exp \left(4 \pi i t P_{n}\right) & \text { for } 0 \leqq t \leqq \frac{1}{2} \\ \left(\exp \left(\frac{4 \pi i}{n}(1-t) Q_{S}\right) \exp \left(\frac{4 \pi i}{n}(1-t) Q_{T}\right)\right)^{n} & \text { for } \frac{1}{2} \leqq t \leqq 1\end{cases}
$$

Then

$$
\left[\rho_{S, T}\right]=\left[\sigma_{S}\right]+\left[\sigma_{T}\right]-\left[\nu_{n}\right] .
$$

On the other hand, for $n_{0}$ sufficiently large and $n \geqq n_{0}$ one has

$$
\left[\rho_{S, T}\right]=\left[\sigma_{S+T}\right] \text {. }
$$

Using lemma 3 one obtains from the last two relations

$$
(+) \varphi(S+T)-\varphi(S)-\varphi(T)=\varphi\left(Q_{S+T}\right)-\varphi\left(Q_{S}\right)-\varphi\left(Q_{T}\right)-\left[\nu_{n}\right]_{\infty} \text {. }
$$

The generalized addition theorem of the exponential function implies

$$
\lim \left\|Q_{S+T}-P_{n}\right\|=0
$$

hence

$$
\nu_{\infty}(t):=\lim \nu_{n}(t)
$$

exists for each $t$ in the norm topology. It follows that $\nu_{\infty}=\lim \nu_{n}$ also uniformly in $t$. One has

$$
\nu_{\infty}(t)=\left\{\begin{array}{l}
\exp \left(4 \pi i t Q_{S+T}\right) \\
\exp \left(4 \pi i(1-t)\left(Q_{S}+Q_{T}\right)\right) .
\end{array}\right.
$$

Lemma 3 implies that

$$
-\left[\nu_{\infty}\right]_{\infty}=\varphi\left(Q_{S}+Q_{T}\right)-\varphi\left(Q_{S+T}\right) .
$$


For sufficiently large $n$ one has

$$
\left[\nu_{\infty}\right]_{\infty}=\left[\nu_{n}\right]_{\infty} .
$$

Together with $(+)$ the last two relations imply lemma 4 .

Lemma 5. Let $S$ satisfy the hypotheses of corollary 3 of theorem 7. Then for each $T \in 1 / c . \mathscr{F}_{c S}$ one has

$$
\varphi(S+T)=\varphi(S)+\varphi(T) .
$$

Proof. One has $\exp (2 \pi i c S)=I$ so that $Q_{c S}=0$. For $c T \in \mathscr{F}_{c S}$ one has $(c S, c T) \in I I$. Hence lemma 4 implies

$$
\begin{aligned}
\varphi(S+T)-\varphi(S)-\varphi(T) & =\frac{1}{c}(\varphi(c S+c T)-\varphi(c S)-\varphi(c T)) \\
& =\frac{1}{c}\left(\varphi\left(0+Q_{c S}\right)-\varphi(0)-\varphi\left(Q_{c T}\right)\right) \\
& =0,
\end{aligned}
$$

THEOREM 8. For each pair $(S, T)$ of positive elements of $\mathscr{M}$ one has

$$
\varphi(S+T)=\varphi(S)+\varphi(T) .
$$

Proor. Let $S \in \mathscr{M}_{+}$. Choose a sequence $\left(S_{k}\right)$ satisfying the hypotheses of corollary 4 of theorem 7 . By lemma 2 all sets $\mathscr{F}_{k}:=\left(1 / c_{k}\right) \mathscr{F}_{c_{k} S_{k}}$ are open dense in $\mathscr{M}_{+}$in the norm topology. Hence their intersection

$$
\mathscr{F}=\bigcap_{k=1}^{\infty} \mathscr{F}_{k}
$$

is norm dense in $\mathscr{M}_{+}$by the Baire category theorem. By lemma 5 one has for each $k$ and each $T_{0} \in \mathscr{F}$

$$
\varphi\left(S_{k}+T_{0}\right)=\varphi\left(S_{k}\right)+\varphi\left(T_{0}\right) .
$$

Since $\lim \left\|S-S_{k}\right\|=0$ and $\varphi$ is norm continuous, this implies

$$
\varphi\left(S+T_{0}\right)=\varphi(S)+\varphi\left(T_{0}\right)
$$

for all $T_{0} \in \mathscr{F}$. Together with the continuity of $\varphi$ and the density of $\mathscr{F}$ this relation implies theorem 8 .

\section{LITERATURE}

1. H. Araki, M.-S.B. Smith and L. Smith, On the homotopical significance of the type of von Neumann algebra factors, Commun. Math. Phys. 22 (1971), 71-88

2. M.F. Atiyah, $K$-Theory, Benjamin, New York 1967 MR $36 \$ 7130$.

3. N. Bourbaki, Elements de mathématique, Fasc. XXXVII, Groupes et algébres de Lie, Chap. III, Hermann, Paris 1972. 
4. M. Breuer, On the homotopy type of the group of regular elements of semifinite von Neumann algebras, Math. Ann. 185 (1970), 61-74 MR 41 \$9003.

5. - Theory of Fredholm operators and vector bundles relative to a von Neumann Algebra, Rocky Mountain J. of Math. 3 (1973), 383-429.

6. M. Breuer, H.O. Cordes, On Banach algebras with $\sigma$-symbol II, J. Math. Mech. 14 (1965).

7. J. Brüning-W. Willgerodt, Eine Verallgemeinerung eines Satzes von N. Kuiper, Math. Ann. 220 (1976) 47-58.

8. J. Dixmier, Les algébres d'opérateurs dans l'espace hilbertien. Paris: GauthierVillars 1957 MR $20 \sharp 1234$.

9. K. Jänich, Vektorraumbündel und der Raum der Fredholm-Operatoren, Math. Ann. 161 (1965), 129-142. MR $32 \$ 8356$.

10. F. J. Murray and J. von Neumann, On rings of operators, Ann. of Math. 37 (1936), 116-229.

11. R. Palais, On the homotopy type of certain groups of operators, Topology 3 (1965), 271-279.

Fachbereich Mathematik, Universität Marburg, 3550 Marburg/Lahn, GerMANY. 